POS $\quad$ PROCEEDINGS

\title{
Micro Pattern Gas Detector Technologies and Applications - The work of the RD51 Collaboration
}

\author{
S. Dalla Torre \\ INFN, Sezione di Trieste, Trieste, Italy \\ E-mail: Silvia.DallaTorreats.infn.it
}

Driven by the availability of modern photolithographic techniques, the Micro Pattern Gas Detectors have been introduced in the last years of the $20^{\text {th }}$ century by pioneer activities: Gas Electron Multipliers (GEM) and Micromegas, later followed by thick-GEM, resistive GEM (RETGEM) and novel micro-pattern devices. Nowadays, a flourishing of R\&D activities dedicated to MPGDs and of diversified applications is ongoing, largely favored by the technological collaboration RD51. The collaboration mission is to facilitate the development of these advanced gas-avalanche detector technologies and associated electronic-readout systems, to be used in basic and applied research. The areas of activities within RD51 include MPGD technology and new structures, device characterization, software and simulations, electronics, MPGD production, common test facilities, and applications of MPGDs. By this coverage of all aspects of MPGDs, RD51 brings together the leading experts in the field of MPGD developments and MPGD users in a wide array of applications.

The RD51 achievements are reviewed by summarising the first five years of the Collaboration activity and by anticipating the future programmes, planned over the next five years.

Technology and Instrumentation in Particle Physics 2014,

2-6 June, 2014

Amsterdam, the Netherlands 


\section{Historical introduction}

Gaseous detectors of ionising particles have accompanied the progress of nuclear and particle physics: since the introduction in 1908 of the Geiger Counter [1]. In 1968, the Multi-Wire Proportional Chambers (MWPC) [2] provided for the first time fine space resolution. Still today gaseous detectors offer unique opportunities: they provide the only possibility to equip large volumes at a reasonable cost, operating in magnetic field with good space resolution and limited material budget. Resistive Plate Chambers (RPC) [3] present the time resolution record in extended counters: they are used in the trigger systems of ALICE, ATLAS and CMS at CERN LHC.

The first Micro-Pattern Gaseous Detector (MPGD) architecture is the Micro-Strip Gas Chamber (MSGC) [4], designed for fine space resolution and large rates. Unfortunately, the high electric field at electrode edges was causing damages to the detector and the charge accumulation at the insulator surface was the source of an important gain evolution versus time. About ten years later, the passivation of the cathode edges was introduced: these detectors have been adopted in high-energy physics experiments and low energy applications.

In the late 90's, two new MPGD architectures, both intended to improve space resolution and rate capabilities, were introduced: Micromegas [5] and Gas Electron Multipliers (GEM) [6]. The first large scale application within the COMPASS experiment [7] at CERN SPS established these detector types and provided reference performance in an experimental environment: Micromegas detectors exhibit a time resolution of $9 \mathrm{~ns}$ and a space resolution of $90 \mu \mathrm{m}$, GEM-detectors a time resolution of $12 \mathrm{~ns}$ and a space resolution of $70 \mu \mathrm{m}$.

The detector discharge rate represents the most critical issue for the reliability of the MPGDs. Concerning Micromegas, early studies [8] indicate that, in efficiency plateau conditions, a variation of the applied HV of $10 \%$ can cause an increase of the discharge probability of three orders of magnitude and more. In GEM-detectors, the control of the discharge probability has been obtained by multi-layer architectures: for a given gain-value, the discharge probability in triple GEM-detectors is more than five orders of magnitude lower than in single GEM-detectors [9].

The CERN-based technological collaboration RD51 [10], dedicated to facilitate the progress and dissemination of MPGDs in High Energy Physics (HEP), in science in general and in applications beyond science, started its activity at the end of 2008. The remarkable progress in the MPGD field registered in the last years is largely based on the RD51 work. In the following, RD51 is described (Sec. 2) as well as the consolidation of the established architectures (Sec. 3), the novel MPGD architectures (Sec. 4) and examples of the wide MPGD application portfolio (Sec. 5).

\section{The RD51 Collaboration}

The RD51 Collaboration, originally foreseen for a first term (2009-2013), has been recently approved for a five-year prolongation, namely till the end of 2018, thanks to its successful results. Presently about 90 Institutions from four continents, including a community of 500 physicists, participate to RD51. The Collaboration serves as an access point to the MPGD know-how for the world-wide community and it provides crucial tools for the detector progress and the applications.

RD51 activity is organised in seven Work Packages (WP), where WPs 1,2, 4 and 7 are mainly dedicated to progress in the MPGD domain, while WPs 3, 5 e 6 are meant to facilitate the dis- 
semination of the technologies (more information about WPs 1-3 is provided in Secs. 3, 4 and 5):

- Technological Aspects and Development of New Detector Structures (WG1).

- Common Characterisation and Physics Issues (WG2).

- Applications (WG3).

- Simulations and Software Tools (WG4): In this WG, a common, open-access, maintainable software suited for the simulation of MPGD detectors is developed, staring from the original Garfield [11]. Thanks to the RD51 support, the package is maintained, continuously updated and enriched of ingredients specific to the MPGDs, as, for instance, the precise description of the electrostatic configurations when dramatic electric field variation take place within microscopic distances comparable with the electron mean free path, as it is the case in MPGDs, and the role of the open dielectric surfaces present in the majority of the MPGDs.

- MPGD related electronics (WG5): The Scalable Readout System (SRS) [12] developed within RD51 as part of the collaboration mission, offers a modular readout architecture providing common hardware elements and software tools with the capability to interface different front-end chips. The cost is moderate ranging around 2 euros per channel without including the front end components. Such a system is highly needed by the detector developers, who were missing a compact, modular read-out system available off-the-shelf. Nevertheless, its scalability has led to consider this readout system for the ATLAS MAMMA project, where 100 thousand channels are required. Thanks to its flexibility, it has been considered also for the read-out of detectors outside the MPGD family.

- Production (WG6): In this working group cost-effective, industrial technology solutions are developed and transferred to industry; a common production facility, based on the MPGD workshop at CERN is being developed and maintained and procedures for industrialisation are set up here. The wide development of the MPGD would not be possible without the presence at CERN of a reference workshop, originally established for PCB production [13]. The development of GEMs, multiplier foils and read-out elements, took fundamental advantage from the technological support of this workshop since the very beginning (1996); a few years later Micromegas components were produced there, soon followed by the production of complete detectors by a technique developed with the fundamental contribution of the workshop: the bulk Micromegas. The role of the workshop is central also in the development of the resistive Micromegas. The development towards large size detectors, both in the GEM and in the Micromegas sector, could take place thanks to the workshop effort. A number of different structures (Sec. 4) are produced there. Thank to the context created by RD51, an enlarged and optimised site for the workshop is being completed with enriched equipment for large-size detector production. Concerning the effort to transfer the production of crucial detector components to industry, it involves four companies for GEM-foil production, two for Micromegas and two for THGEMs. 
- Common Test Facilities (WG7): A common, wide, richly equipped laboratory located at CERN and a common test beam area at CERN SPS are maintianed and several research groups within RD51 take advantages of these facilities.

The RD51 programme for the second activity term, just started, is based on the continuation of the present activity, with special emphasis to the completion of the technology transfer for the MPGD industrial production and to the dissemination actions in order to fully exploit all the MPGD potentiality in a wide portfolio of applications.

\section{The consolidation of the established architectures}

In Micromegas, the multiplication is provided by the parallel plate electrode structure operated in avalanche mode over a very thin gap, of the order of $100 \mu \mathrm{m}$. The cathode has a micromesh structure to let the ionisation electrons produced outside the multiplication gap enter the gap region. This geometry has imposed construction difficulties since the realisation of the very first prototypes. Initially electroformed Ni mesh were used, while the spacing between the mesh and the anode was ensured by quartz fibres of $75 \mu \mathrm{m}$ diameter. The fibres introduced non negligible dead zones. A few years later, novel micromesh were used formed by etched metallised polyimide foils equipped with micropillars produced by a photolitographic process and acting as spacers [14]. This process is chosen for the large chambers required by the Muon ATLAS MicroMegas Activity (MAMMA) [15]. Bulk Micromegas [16] are obtained by pre-stretching a steel micromesh, which is then laminated together with the PCB forming the detector anode and a photoresistive layer sandwiched between the mesh and the PCB plate. The largest portion of the photoresistive material is then removed and only the portion forming the distance micropillars is left. A robust detector is obtained, which, however, cannot be dismounted for cleaning and maintenance.

Concerning the handling of the discharge rates, two recent approaches are of interest. In the context of the MAMMA project, resistive anodes have been introduced [17] to limit the discharge energy, which results in a protection of the detector itself and of the front-end electronics and, even more relevant, in a substantial reduction of the detector dead-time; in fact, a non negligible dead-time is present at high discharge rates due to the time required to re-establish the operational high voltage after a discharge event. For the high rate environments of the experiments COMPASS and CLAS12 at Jefferson Lab CEBAF, a reduction of one order of magnitude and more is obtained thanks to a GEM pre-amplification stage [18].

Pixelised Micromegas are under development for the COMPASS experiment, designed to cope with the high flux of the beam region [19].

The gain at which the detectors are operated in the experiments is low: for instance it is about 6400 for the trackers in use at COMPASS [7] and about 1500 for the TPC read-out detectors at T2K [20] neutrino experiment at Tokai. Concerning rate capabilities, prototypes of the resistive Micromegas for the MAMMA project preserve a linear response up to a hit rate of $100 \mathrm{kHz} / \mathrm{cm}^{2}$ [15]. The material budget of a standard Micromegas is $0.3 \%$ of a radiation length [7]. Detailed ageing studies have been performed in the context of the MAMMA project irradiating resistive Micromegas prototypes with a variety of different ionising particles [21]: no response degradation has been observed. 
The construction of the GEM-detectors implies two main issues: the GEM-foil production and the preservation of the correct spacing between the GEMs in multilayer counters. The GEMfoils are produced by chemical etching of metallised polyimide foils and a better and better control of the process has been established to avoid geometrical variations causing different detector performance [22]. GEM foils produced by laser hole formation is also attempted [23, 24]. Initially, the GEM foils were etched using a double mask approach: the chemical attack was active from both foil faces. The difficulty of a perfect alignment of the two masks over large surfaces suggests a single mask production protocol, initially developed for the upgrade of the TOTEM experiment at CERN LHC [25], then used to produce the GEM foils for the KLOE2 cylindrical GEM-detector [26], foreseen for the CERN LHC CMS forward muon spectrometer [27].

At first, the correct spacing between the GEM foils in multilayer detectors of large-size was obtained by adequate spacers, which introduce dead zones (the COMPASS [28] and TOTEM trackers [29]). For the trackers of LHCb at CERN LHC, the INFN groups introduced an alternative approach: the foil stretching [30]. The foils are pre-stretched and then glued onto their support frames. This construction concept opened the way to the cylindrical GEM-detector of the experiment KLOE-2 at the LNF DAFNE - $\Phi$ factory [26]. More cylindrical GEM-detectors are expected: CMD-3 [31] at the Budker INP VEPP-2000 Collider and BESIII [32] at the Beijing ElectronPositron Collider. The gigantic production foreseen for the CMS forward muon spectrometer [27] is based on an extension of the stretching approach: the mechanical stretching without gluing in order to save on assembly time.

A very fine time resolution of about $4.5 \mathrm{~ns}$ has been obtained for the LHCb detectors, optimising in view of this achievement the geometrical parameters and the gas mixture [33]. The gain at which the triple layer detectors are operated in experiments is low: for instance it is about 4000 for the LHCb trackers [30], about 8000 for the TOTEM trackers [34], and about 4000 for the PHENIX HBD photon detectors [35]. The material budget of a standard triple GEM detector is $0.4 \%$ of a radiation length [7], while it has been reduced, mainly by a thinner $\mathrm{Cu}$ coating of the polyimide foils, to about $0.2 \%$ of a radiation length in the pixel GEM [36] in operation at the COMPASS experiment. Concerning rate performance, this pixel-GEM can successfully operate at rates as high as $1.2 \times 10^{5} / \mathrm{mm}^{2} / \mathrm{s}$. Systematic studies about GEM-tracker ageing have been performed for the LHCb detectors: at high gas fluxes no degradation is observed up to integrated charges as large as $2 \mathrm{C} / \mathrm{cm}^{2}$ [37]. The successful operation of GEM-detectors in cryogenic environment has been proved [38].

THGEMs, introduced in parallel by several groups [39, 40], are electron multipliers derived from the GEM design, scaling the geometrical parameters and changing the production technology. The Cu-coated polyimide foil of the GEM multipliers is replaced by standard Printed Circuit Boards (PCB) and the holes are produced by drilling. The conical shape of the GEM holes that forms uncoated polyimide rings around the holes themselves is replaced by a clearance ring, the rim, surrounding the hole and obtained by $\mathrm{Cu}$ etching. Large gains are reported, both using THGEMs in single and multiple arrangements. THGEMs have an intrinsic mechanical stiffness, and they are robust against damages. They can be produced in large series and large size, in spite of the large number of holes: some millions per square meter. Due to the geometrical dimensions which are about one order of magnitude larger than in GEMs, the detector has specific features, where the electron diffusion plays a less relevant role respect to GEMs and the coupling between 
the dipole field across a layer and the transfer field between layers is definitively stronger. The first phase of the THGEM characterisation was almost entirely performed by the Weizmann group leaded by A. Breskin [41]: this effort resulted in a deep knowledge of the response dependence versus the geometrical parameter of the multipliers, the gas mixture and pressure and the overall multilayer detector architecture. Further characterisation studies, mainly aiming at the design and realisation of novel photon detectors for Cherenkov imaging applications, have been performed in view of the upgrade of COMPASS RICH-1 [42, 43, 44]; in particular, the evolution of the gain response versus time has been clarified and architecture modifications in order to reduce the ion back-flow to the photocathode have been investigated. Recently, THGEM engineering aspects have received attention. In general, a large fraction of the industrially produced THGEMs cannot reach the maximum voltage expected according to gas and geometry. A polishing procedure has been introduced [42, 44]: it makes always possible to rise the breakdown voltage value of largesize THGEMs above $90 \%$ of the maximum expected value. The uniformity of the gain response in large size THGEMs has been related to the uniformity of the board thickness and satisfactory results are obtained by plate pre-selection.

\section{The novel MPGD architectures}

Novel architectures mainly derived from the GEM and Micromegas structures have been introduced to answer to specific requirements.

A family of GEM-derived detectors obtained adopting the production technology already developed for the GEM multipliers is represented by the Micro-Hole and StriP plates (MHSP) [45] and the Cobra multipliers [46]. Extra electrodes are introduced at a face of the multiplier plate in order to modify the electric field lines: the ion trapping at the intermediate layers of a multi-layer detector architecture is enhanced and rates of the ion back-flow to the photocathodes as low as $10^{-4}$ and less can be obtained. These multipliers are developed for MPGDs of visible photons. Due to the fragility of the visible solid state photoconverters, visible photon detectors are often sealed ones: the use of non out gassing materials is a relevant issue and borosilicate GEMs, where holes are produced by a micro-blasting technique, are under development [47, 48]; they are derived from the capillary plate concept [49]. In this context, the availability of photo-etchable glass and its use to produce glass GEMs by etching technology [50] is a novel approach of interest.

Resistive GEMs (Re-GEM) [51] consisting in GEMs with electrodes by resistive polyimide are considered to reduce the damages due to the detector discharges for applications at high rate and large gain.

The Piggy Back detector [53] is derived from the Micromegas structure replacing the segmented conductive anode plane by a three layer sandwich: a resistive anode, a ceramic insulator and a segmented metallic read-out layer. The high resistivity of the anode plane and the capacity coupling between the anode and the read-out electrodes form a high-pass filter. The fast signals are transmitted with minimal losses. The resistive layer reduces the energy release in case of discharges. The read-out elements are fully separated from the active volume.

In the micro-bulk Micromegas [54] the amount of material budget is reduced and radioactive pure materials are selected for fundamental research in the rare event sector. 
The goal of the GRIDPIX detector [55] is a space resolution similar to that of a silicon pixel detector obtained by a gaseous detector able to detect single electrons. It is obtained by postprocessing of a silicon read-out chip (Timepix [56]) growing a micromesh directly onto the chip itself. Large size prototypes have been recently obtained. This detector aims at the read-out of Time Projection Chambers (TPC) in extremely crowded environments.

The Micro-PIxel Chamber ( $\mu$ PIC) structure [57], produced by making use of the PCB technology, and offering 2D information, follows a geometrical arrangement where no floating electrodes are present: the goal is a robust general purpose detector characterised by reduced production costs, thanks to a fully industrial production process. Recently the detector has been made more spark-tolerant by the introduction of a thin resistive layer covering the cathode electrodes [58].

Important advantages can be obtained by combining elements of the various MPGD architectures in a single detector, or even combining MPGD elements with non gaseous detectors.

The first hybrid detector dates back to the early days of MPGDs: already in 1997 detector schemes obtained combining a GEM multipliers and either a MWPC or a MSGC had been studied [59]. More hybrid structures have been proposed for the photon detection: borosilicate GEM coupled to a Micromegas in sealed gaseous PhotoMultiplier Tubes (PMT), where the Micromegas stage largely contributes in reducing the ion back-flow to the photocathode, and a THGEM multiplication stage followed by a Micromegas [42], where the THGEM is the photoconverter substrate and, again, the Micromegas improves the control of the ion back-flow. The GEMpix detector [60] is formed by a GEM amplification stage followed by a read-out stage based on the medipix chip [61].

\section{The MPGD applications}

MPGDs have been introduced for the needs of fundamental research in the domain of high energy and particle physics and this is the context where they have been validated and improved.

GEM- and Micromegas-trackers are in use at COMPASS [7], at CAST [62], at LHCb [30] and at TOTEM [29]. The read-out elements of the TPC at the T2K experiment is formed by a mosaic of Micromegas chambers [20]. A set of GEM-detectors ensures the read-out of the FOPI spectrometer TPC [63]. MSGCs have been successfully operated at DIRAC [64] at CERN PS and at Hera-B [65] at Desy Hera.

The relevant success of the applications mentioned so far and the rich spectrum of consolidation and novel schemes makes MPGDs very appealing for future applications in fundamental research. Large size projects are foreseen for the upgrade of all the major CERN LHC experiments. Large-size resistive Micromegas are foreseen to equip the new small wheel of the ATLAS muon system for tracking and triggering within the MAMMA project [15], presently approved; it foresees chambers of a few $\mathrm{m}^{2}$ each for a total surface of about $1200 \mathrm{~m}^{2}$. The CMS experiment plans to equip the forward muon spectrometer with large-size GEM detectors [27] for tracking and triggering with a total detector surface of about $300 \mathrm{~m}^{2}$. GEM foils for a total surface of almost $1000 \mathrm{~m}^{2}$ are needed. The construction of a first portion of the overall system has been approved. The ALICE experiment has recently approved the upgrade of the TPC read-out system [66] aiming at replacing the present MWPCs with GEM-detectors, about $1 \mathrm{~m}^{2}$ wide, for a total production of $130 \mathrm{~m}^{2}$ of GEM foils. The gigantic production of MPGD components required by these extended 
projects points to the absolute need of transferring the mass production to industry, ensuring reliable production (Sec. 2).

GEM detectors are also proposed for tracking at the JLab Hall A experiment [67], for the upgrade of the Forward tracker at the BNL RHIC STAR experiment [68], as active elements for the hadron sampling calorimetry [69] at the future International Linear Collider (ILC) and as TPC readout elements [70] at ILC. THGEM are proposed as an alternative to GEM for the hadron sampling calorimetry [71] at ILC. Micromegas are considered for tracking in the forward region [72] and in the vertex region with cylindrical geometry [73] at the JLAB CLAS12 experiment and as TPC read-out elements at ILC [74].

MPGDs are crucial elements in a number of future non-accelerator experiments: THGEMs are considered for LBNO-Glacier [75] dedicated to neutrino detection and for the dark matter experiment XENON-100 [76], hybrid MPGD coupling THGEMs and Micromegas are developed for dark matter searches with the Panda-X experiment [77], while Micromegas are present in the design of NEXT-100 [78] dedicated to neutinoless double beta decay and IAXO [79] for axion searches.

In particle and nuclei experimental studies, gas detectors of single photons have been mainly developed for the application in Cherenkov imaging counters, where, still nowadays, they represent the only available option to instrument detection surfaces when insensitivity to magnetic field, low material budget, and affordable costs in view of large detection surfaces are required. MPGD-based photon detectors represent novel architectures able to offer two relevant advantages: intrinsically fast signals and reduced photon and ion back-flow to the photocathode. The first studies of photon detection by MPGDs date back to the end of the previous century and are based on the use of GEM detectors [80], while the first large-scale successful application is the Cherenkov threshold Hadron Blind Detector (HBD) [35] of the RHIC PHENIX experiment. THGEM-based photon detectors are being developed for the ALICE Very High Momentum Particle IDentification (VHMPID) [81] and are under construction for the upgrade of the COMPASS RICH-1 photon detection system [42]: the present MWPC photon detectors will be replaced by hybrid THGEM-Micromegas detectors equipped with CsI photocathodes.

The panorama of applications outside high energy and particle physics is rich and it can be quoted only by examples, aiming to offer a clear indication that the transfer of the MPGD knowhow to sectors different from the original is ongoing. The large MSGC setup at the D20 diffractometer [82] at ILL in Grenoble is an example of application at an intense neutron source. GEMdetectors coupled to adequate neutron converters are used to monitor neutron beams [83]. A TPC with GEM read-out to be used as X-ray polarimeter is in preparation for the NASA GEMS mission [84], where effective X-ray detection is required. A TPC equipped with GEM read-out is under development for the superconducting in flight separator for exotic nuclei up to relativistic energies Super-FRS at the future GSI FAIR facility [85]. This project tests the MPGD potentialities for the diagnostic of high intensity beams. The NIFFTE TPC with Micromegas read-out [86] and the active target with GEM read-out for the study of deuteron inelastic scattering off exotic nuclei [87] under development at NSC, Tokyo, exemplify the application to low energy nuclear studies. The feasibility of fast-neutron tomography by THGEM-detectors as diagnostic in nuclear plants is the goal of an intense R\&D development [88]. A TPC with two layers of Micromegas read-out is studied for muon radiography dedicated to geological studies in the context of the Tem- 
poral Tomography by the Measure of Muons (TTDM2) project [89].

\section{Conclusions and outlook}

The impressive progress and the great potentiality of the MPGDs have been illustrated by examples in previous sections. In this context, the role played by the RD51 Collaboration is central. A coherent community of MPGD developers and users has been created: the information exchange within RD51 is open, continuous and diversified, favouring both the developments and the applications, while more support to the world-wide community is offered by the infrastructure and tools provided within RD51. RD51 is also stimulating the dissemination of MPGDs beyond the context where they have been introduced, namely beyond HEP, towards the usage in other science fields and in applications in sectors of social interest. RD51 dedicates a special effort to implement the industrial production of MPGD components: a must for large-size systems as required in frontier HEP experiments and an absolute pre-requisite for the applications beyond science.

\section{References}

[1] E. Rutherford and H. Geiger, Proceedings of the Royal Society (London), Series A 81, (1908) 141.

[2] G. Charpak et al., Nucl. Instr. and Meth. 62 (1968) 235.

[3] R. Santonico and R. Cardarelli. Nucl. lnstr. and Meth. 187 (1981) 377; R. Cardarelli et al., Nucl. Instr. and Meth. A 263 (1988) 20.

[4] A. Oed, Nucl. Instr. and Meth. A 263 (1988) 351

[5] Y. Giomataris et al, Nucl. Instr. and Meth. A 376 (1996) 29.

[6] F.Sauli, Nucl. Instr. and Meth. A 386 (1997) 531.

[7] P. Abbon et al., COMPASS Collaboration, Nucl. Instr. and Meth. A 577 (2007) 455.

[8] D. Thers et al., Nucl. Instr. and Meth. A 469 (2001) 133.

[9] S. Bachmann et al., Nucl. Instr. and Meth. A 479 (2002) 294.

[10] The RD51 Collaboration, R\&D proposal, Development of MicroPattern Gas Detectors Technologies, RD51 2008-001, 28 July 2008, CERN-LHCC-2008-011 / LHCC-P-001 14/08/2008.

[11] R. Veenhof, Nucl. Instr. and Meth. A419 (1998) 726.

[12] S. Martoiu et al., JINST 8 (2013) C03015.

[13] R. De Olivera, "Fabrication techniques and industrialization of MPGDs", invited talk at third Conference on Micro-Pattern Gaseous Detectors (MPGD2013), 1-4 July 2013, Zaragoza, Spain.

[14] A. Delbart et al., Nucl. Instr. and Meth. A 461 (2001) 84.

[15] T. Alexopoulos et al., Nucl. Instr. and Meth. A 617 (2010) 161; G. Iakovidis et al., JINST 8 (2013) C12007.

[16] Y Giomataris et al., Nucl. Instr. and Meth. A 560 (2006) 405.

[17] J. Manjarrés et al., JINST 7 (2012) C03040; T. Alexopoulos, G. Iakovidis and G. Tsipolitis, JINST 7 (2012) C05001. 
[18] S. Procureur et al., Nucl. Instrum. Meth. A 659 (2011) 91.

[19] M. Vandenbroucke, Development and Characterization of Micro-Pattern Gas Detectors for Intense Beams of Hadrons, PhD thesis, Université Pierre et Marie Curie and Technische Universität München, 2012.

[20] N. Abgrall et al., Nucl. Instr. and Meth. A 637 (2011) 25.

[21] J. Galan et al., Ageing studies of resistive Micromegas detectors for the HL-LHC, 13th Vienna Conferemnce on Instrumentation (VCI2013), 11-15 February 2013, Vienna, Austria.

[22] W. Anderson et al., Understanding the gain characteristics of GEMs inside the Hadron Blind Detector in PHENIX, 2007 IEEE Nuclear Science Symposium Conference Record, NSS ’07. IEEE, IEEE, Piscataway, NJ, USA, 2008, pp. 4662.

[23] T.Tamagawa et al., Nucl. Instr. and Meth. A 560 (2006) 418; T.Tamagawa et al., "GEM-TPC X-ray polarimeter onboard GEMS satelite", third Conference on Micro-Pattern Gaseous Detectors (MPGD2013), 1-4 July 2013, Zaragoza, Spain. JINST 9 (2014) C01002.

[24] V. Berardi, Laser drilling of a copper mesh, 7th RD51 Collaboration meeting, 13-15 April 2011, CERN.

[25] M. Villa et al., Nucl. Instr. and Meth. A 628 (2011) 182.

[26] A. Balla et al., Nucl. Instr. and Meth. A 628 (2011) 194. G. Morello et al., "The Cylindrical-GEM detectors for the KLOE-2 Inner Tracker", JINST 9 (2014) C01014.

[27] D. Abbaneo et al., Nucl. Instr. and Meth. A 718 (2013) 383; M. Tytgat et al., JINST 8 (2013) C12031.

[28] B. Ketzer et al., Nucl. Instr. and Meth. A 535 (2004) 314.

[29] M.G. Bagliesi et al., Nucl. Instr. and Meth. A 617 (2010) 134.

[30] M. Alfonsi et al., Nucl. Instr. and Meth. A 518 (2004) 106; M. Alfonsi et al., Nucl. Instr. and Meth. A 581 (2007) 283.

[31] L. Shekhtma et al., JINST 8 (2013) C12035.

[32] Workshop on the Cylindrical GEM Inner Tracker for BESIII, Frascati, Italy, 17 April 2013, https://agenda.infn.it/conferenceDisplay.py?confId=6213.

[33] M. Alfonsi et al., Nucl. Instr. and Meth. A 535 (2004) 319.

[34] G. Catanesi, private communication.

[35] W. Anderson et al., Nucl. Instrum. Meth. A 646 (2011) 35.

[36] M. Kramer et al., Nuclear Science Symposium Conference Record, 2008. NSS '08. IEEE, IEEE, Piscataway, NJ, USA, 2008, pp. 2920; A. Austregesilo et al., Nucl. Phys. B (Proc. Suppl.) 197 (2009) 113.

[37] M. Alfonsi et al, Nucl. Phys. B Proc. Suppl. 150 (2006) 159.

[38] A. Bondar et al., Nucl. Instr. and Meth. A 581 (2007) 241.

[39] R. Chechik, A. Breskin, C. Shalem and D. Mormann, Nucl. Instrum. Meth. A 535 (2004) 303.

[40] L. Periale et al., Nucl. Instr. and Meth. A 478 (2002) 377; P. Jeanneret, PhD thesis, Neuchatel University, 2001; P.S. Barbeau et al, IEEE NS-50 (2003) 1285.

[41] A. Breskin et al., Nucl. Instr. and Meth. A 598 (2009) 107 and references therein. 
[42] M. Alexeev et al., JINST 8 (2013) C12005.

[43] M. Alexeev et al. Nucl. Instr. and Meth. A 610 (2009) 174; M. Alexeev et al. Nucl. Instr. and Meth. A 617 (2010) 396; M. Alexeev et al., JINST 5 (2010) P03009; M. Alexeev et al., Nucl. Instr. and Meth. A 639 (2011) 130; M. Alexeev et al., Nucl. Instr. and Meth. A 695 (2012) 159; M. Alexeev et al., JINST 7 (2012) C002014; M. Alexeev et al., Nucl. Instr. and Meth. A 623 (2010) 129; M. Alexeev et al., JINST 8 (2013) P01021.

[44] M. Alexeev et al., JINST 9 (2014) C03046.

[45] J.F.C.A. Veloso et al., Rev.Sc. Instr. 71 (2000) 2371; A.V. Lyashenko et al., JINST 2 (2007) P08004.

[46] A.V. Lyashenko et al., Nucl. Instr. and Meth. A 598 (2009) 116.

[47] F. Tokanai et al., NIMA 610 (2009) 164.

[48] F. Tokanai et al., NIMA 628 (2011) 190.

[49] V. Peskov et al., Nucl. Instr. and Meth. A 433 (1999) 492; J.Va'vra and T. Sumiyoshi, Nucl. Instr. and Meth. A 435 (2004) 334.

[50] Y. Mitsuya, JINST 8 (2013) C11018; T. Fujiwara et al., JINST 8 (2013) C11018.

[51] A. Yoshikawa et al., JINST 7 (2012) C06006.

[52] J.M. Bidault et al., Nucl. Phys. B 158 (Proc. Suppl.) (2006) 199; R. Oliveira et al., /http://arxiv.org/abs/physics/0701154S; G. Agocs et al., JINST 3 (2008) P02012; G. Agocs et al., Nucl. Instr. and Meth. A 595 (2008) 128; A. Di Mauro et al., Nucl. Instr. and Meth. A 581 (2007) 225.

[53] D Attié et al., JINST 8 (2013) P05019.

[54] S. Andriamonje et al., JINST 5 (2010) P02001.

[55] M. Chefdeville et al., Nucl. Instr. and Meth. A 556 (2006) 490; M. Lupberger, JINST 9 (2014) C01033; W.J.C. Koppert et al., Nucl. Instr. and Meth. A 732 (2013) 1.

[56] X. Llopart et al., Nucl. Instr. and Meth. A 581 (2007) 485.

[57] A. Ochi et al., Nucl. Instr. and Meth. A 471 (2001) 264.

[58] A. Ochi et al., JINST 9 (2014) C01039.

[59] R. Bouclier et al., Nucl. Instr. and Meth. A 396 (1997) 50.

[60] A. Bamberger et al., Nucl. Instr. and Meth. A 581 (2007) 274.

[61] A. Bamberger, et al., Nucl. Instr. and Meth. A 573 (2007) 361.

[62] S. Aune et al., Nucl. Instr. and Meth. A 573 (2007) 455.

[63] S. Dørheim et al., JINST 7 (2012) C03011; F. V. Boehmer, "Performance of a Large GEM-TPC at FOPI", third Conference on Micro-Pattern Gaseous Detectors (MPGD2013), 1-4 July 2013, Zaragoza, Spain.

[64] B. Adeva et al., Nucl. Instr. and Meth. A 515 (2003) 467.

[65] T. Hott, for the Hera-B inner tracker collaboration, Nucl. Instr. and Meth. A 408 (1998) 258.

[66] M. Ball et al., "GEM upgrade of the ALICE TPC" third Conference on Micro-Pattern Gaseous Detectors (MPGD2013), 1-4 July 2013, Zaragoza, Spain.

[67] V. Bellini et al., JINST 7 (2012) C05013; E. Basile et al., Nucl. Instr. and Meth. A 718 (2013) 429. 
[68] B. Surrow, Nucl. Instr. and Meth. A 617 (2010) 196.

[69] A. White, J. Yu, S. Park, Journal of Physics: Conference Series 404 (2012) 012031.

[70] Bernhard Ledermann et al., IEEE TNS 53 (2006) 2936.

[71] L. Arazi et al., JINST 7 (2012) C05011; S. Bressler et al., . JINST 1307 (2013) P07017.

[72] G. Charles et al., "Progress on CLAS12 Micromegas detectors", third Conference on Micro-Pattern Gaseous Detectors (MPGD2013), 1-4 July 2013, Zaragoza, Spain.

[73] S. Aune et al., Nucl. Instr. and Meth. A 604 (2009) 53.

[74] D.C. Arogancia et al., Nucl. Instr. and Meth. A 602 (2009) 403.

[75] A. Badertscher et al., Nucl. Instr. and Meth. A 641 (2011) 48.

[76] F.Balau et al., Nucl. Instr. and Meth. A598 (2009) 126; A. Breskin et al., JINST 7 (2012) C06008.

[77] S. Duval et al., Nucl. Instr. and Meth. A 695,(2012) 163.

[78] F. J. Iguaz et al., JINST 6 (2011) P07002.

[79] J. Gracia Garz et al., JINST 8 (2013) C12042.

[80] R. Chechwik et al., Nucl. Instr. and Meth. A 419 (1998) 423

[81] A. Di Mauro et al., Nucl. Instr. and Meth. A 639 (2011) 274.

[82] P. Convert et al., Physica B 276-278 (2000) 93.

[83] G. Croci et al., Nucl. Instr. and Meth. A 720 (2013) 144; G. Croci et al., Nucl. Instr. and Meth. A 732 (2013) 217.

[84] T. Tamagawa et al., "GEM-TPC X-ray Polarimeter onboard GEMS Satellite” third Conference on Micro-Pattern Gaseous Detectors (MPGD2013), 1-4 July 2013, Zaragoza, Spain; Y. Takeuchi et al., JINST 9 (2014) C01002.

[85] H. Geissel et al., Nucl. Instr. and Meth. B 204 (2003) 71; F. García et al., "SuperFRS GEM-TPC Development for the FAIR Facility", third Conference on Micro-Pattern Gaseous Detectors (MPGD2013), 1-4 July 2013, Zaragoza, Spain.

[86] J. Ruz et al., JINST 8 (2013) C12018.

[87] C. S. Lee et al., JINST 9 (2014) C05014.

[88] M. Cortesi et al., JINST 8 (2013) C10009.

[89] P. Salin, “T2DM2: Electronic Status \& Progress”, 9th RD51 Collaboration meeting, 20-22 February 2012, CERN. 